\title{
Anomalous zipping dynamics and forced polymer translocation
}

\author{
A. Ferrantini, E. Carlon \\ Institute for Theoretical Physics, K.U.Leuven, Celestijnenlaan 200D, B-3001 Leuven, \\ Belgium \\ E-mail: alessandro@itf.fys.kuleuven.be, enrico.carlon@fys.kuleuven.be
}

\begin{abstract}
We investigate by Monte Carlo simulations the zipping and unzipping dynamics of two polymers connected by one end and subject to an attractive interaction between complementary monomers. In zipping, the polymers are quenched from a high temperature equilibrium configuration to a low temperature state, so that the two strands zip up by closing up a "Y"-fork. In unzipping, the polymers are brought from a low temperature double stranded configuration to high temperatures, so that the two strands separate. Simulations show that the unzipping time, $\tau_{u}$, scales as a function of the polymer length as $\tau_{u} \sim L$, while the zipping is characterized by anomalous dynamics $\tau_{z} \sim L^{\alpha}$ with $\alpha=1.37(2)$. This exponent is in good agreement with simulation results and theoretical predictions for the scaling of the translocation time of a forced polymer passing through a narrow pore. We find that the exponent $\alpha$ is robust against variations of parameters and temperature, whereas the scaling of $\tau_{z}$ as a function of the driving force shows the existence of two different regimes: the weak forcing $\left(\tau_{z} \sim 1 / F\right)$ and strong forcing $\left(\tau_{z}\right.$ independent of $\left.F\right)$ regimes. The crossover region is possibly characterized by a non-trivial scaling in $F$, matching the prediction of recent theories of polymer translocation. Although the geometrical setup is different, zipping and translocation share thus the same type of anomalous dynamics. Systems where this dynamics could be experimentally investigated are DNA (or RNA) hairpins: our results imply an anomalous dynamics for the hairpins closing times, but not for the opening times.
\end{abstract}

PACS numbers: 82.35.Lr; 36.20.-r; 87.15.Aa

Submitted to: JSTAT

Keywords: zipping, polymer translocation. 


\section{Introduction}

The aim of this paper is to study the zipping and unzipping dynamics of polymers. Zipping occurs when two polymer strands, with attractive interactions between complementary monomers, bind to form a double-stranded conformation. This is the behavior of complementary DNA strands forming a double helical structure by closing up a Y-fork in which two single strands join into a double stranded segment (hence the name zipping). The reverse transition, the unzipping, is the separation of the two strands at high temperatures, which can also occur under the effect of a mechanical force pulling the edges of a polymer. Mechanical unzipping has been the subject of several studies in the past [1, 2, 3, 4, 5, 6], due to its relevance to single molecule experiments (see e.g. [7, 8]). Equilibrium properties of zipping transitions have also been investigated [9, 10, 11, 12]. We restrict ourselves here to the case of zipping induced by attractive interactions between monomers, in absence of mechanical forces. We show that the dynamics is anomalous and that it is characterized by an exponent in agreement with that found in polymer translocation [13].

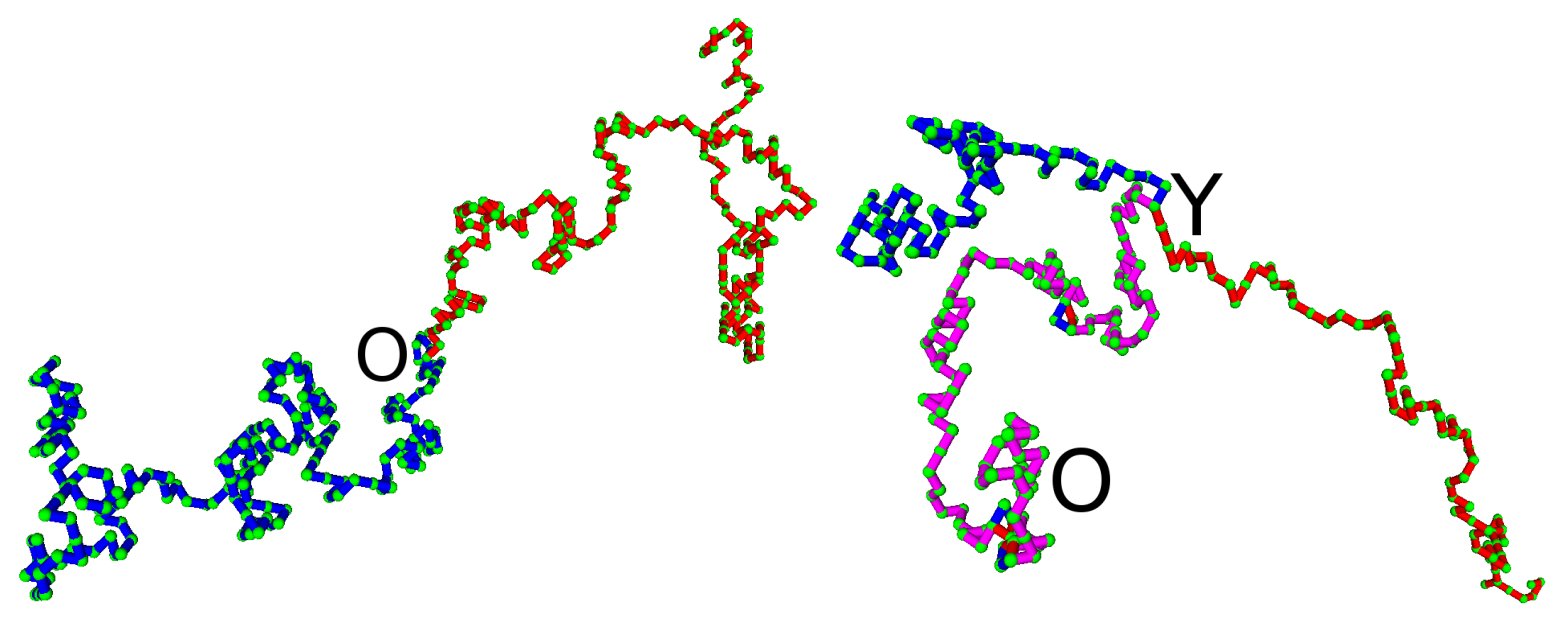

Figure 1. Snapshots of two different polymer configurations (model B, $L=193$, $\omega=0.02$ ). Left panel: an inifinite temperature equilibrium configuration. Right panel: an intermediate configuration during zipping. " $\mathrm{O}$ " denotes the joint end of the two polymers, while "Y" is the branching point where the double-stranded stretch joins the single strands.

In the simulations two polymer strands are attached to each other from one end and are prepared in a high temperature equilibrium state (see Fig. 1, left panel). The system is then quenched to low temperatures, below the thermal unzipping temperature, so that a double-stranded conformation gets formed in the course of time. We consider lattice polymers undergoing Monte Carlo dynamics with local flip moves (see next section for details) which do not violate the self- and mutual avoidance between the strands. This corresponds to a Rouse dynamics, while hydrodynamics effects are neglected. In addition, zipping here occurs without the winding of the strands around each other, as in DNA molecules, but by a pairing of the two strands. The right panel of Figure 1 
shows an example of an intermediate conformation during the zipping process. The branch connecting the points "O" and "Y" is double stranded, and "Y" is the contact point between the zipped part and the single stranded ends. We will also briefly discuss the reverse case of unzipping dynamics where an equilibrated double stranded polymer is brought at high temperatures. This case turns out to be less interesting because the dynamics is not anomalous. However, it provides some insights on the origin of the anomalous dynamics observed during zipping.

Our main interest is the scaling of the zipping time $\tau_{z}$ (to be defined more precisely later) as a function of the length of the strands $L$. Using simulations we find that $\tau_{z} \sim L^{1.37(2)}$. This exponent is in good agreement with that found in the study of a polymer performing a biased translocation through a narrow pore [13]. The same type of scaling has been recently observed in the case of a related problem of polymer adsorption on a flat substrate [14].

Several publications have appeared in the recent literature about translocation of polymers through a pore [15, 16, 13, 17, 18. The pore is a small aperture on a plane which is sufficiently narrow to allow the passage of one or a few monomers at a time. The time needed by the polymer to cross the pore is referred to as translocation time $\left(\tau_{t}\right)$. Although there is a general consensus about the fact that the translocation time scales as a power of the polymer length, the precise numerical value of the exponent has been the subject of some debate [15, 16, 13, 17, 19, 18.

In Ref. [15], then taken up in Ref. [20], a lower bound limit $\tau_{t} \geq L^{1+\nu}$ was derived for the scaling of the translocation time. Here $\nu$ is the Flory exponent $(\nu=0.588)$. This bound was obtained (under some simplifying assumptions) by comparison with the motion of a driven polymer in the bulk, i.e. in absence of the separating plane. Early numerical simulation of the translocation times 21 found a scaling of the type $\tau_{t} \sim L^{1.59}$, particularly in the limit of high pore friction. More recently, Vocks et al. [13] derived the following scaling for the translocation time $\tau_{t} \sim L^{\alpha}$ with $\alpha=(1+2 \nu) /(1+\nu)$, yielding thus $\alpha=1.37$. This prediction is based on memory effects which arise due to an imbalance in the chain tension in the vicinity of the pore and are observed also in the case of unbiased translocation [22]. Numerical simulations [13] confirm the theoretical prediction for $\alpha$. Recent simulations of forced translocation [23] also found $\alpha \approx 1.37$ in a regime referred to as fast translocation.

The adsorption of polymeric chains onto a surface has also been subject of experimental [24, as well as computational and theoretical studies [25, 26, 27, 14]. In the strong adsorption regime, the characteristic adsorption time scales with the chain length as $\tau_{a} \sim L^{1+\nu}[25,26]$. The exponent $1+\nu$ was explained with a two-phase model in which an intermediate adsorbed configuration is viewed as consisting of two nonequilibrium phases: the adsorbate and the non-adsorbed phase (the corona), connected by a stretched part of the chain (the stem). More recent simulations [14] showed two regimes for polymer adsorption, depending on the adsorption energies: $\tau_{a} \sim L^{1.37}$ for weak adsorption energies and $\tau_{a} \sim L^{1.59}$ for strong adsorption energies.

In view of these results, and motivated by the recent interest in anomalous dynamics 
in translocation and adsorption, we investigate here the possibility of observing these effects in the context of polymers zipping. We find indeed anomalous dynamics in the zipping process, but not in the reverse case of unzipping. In the zipping case we investigate the effects of changing temperature and other parameters in the system. Our results show that there is only one regime in this system with a scaling of the zipping time of the type $\tau_{z} \sim L^{1.37}$. Instead, we do not observe a regime with $\tau_{z} \sim L^{1.59}$, as seen in forced translocation [23] and in adsorption [14].

\section{Model}

The model discussed here was also used in a recent study of renaturation dynamics [28], where the scaling properties of the nucleation rates of complementary polymers were investigated. We consider two polymers defined on a face-centered-cubic lattice and joined by one end. We label the monomers of the two strands starting from the common monomer (corresponding to $i=0$ ), with $i=1,2, \ldots L$. The two strands are self- and mutually avoiding, with the exception of monomers with the same index $i$, which are referred to as complementary monomers. Two complementary monomers can indeed bind by overlapping on the same lattice site.

The polymers undergo Rouse dynamics which consists of local corner-flip or endflip moves that do not violate self- and mutual avoidance. The overlap between complementary monomers, which thus form a bound pair, is always accepted as a move. The opposite move, that of detaching two bound complementary monomers, is accepted with probability $\omega=\exp \left(-\varepsilon / k_{B} T\right)$, so that detailed balance is satisfied. Here $\varepsilon>0$ is the binding energy, $k_{B}$ the Boltzmann constant and $T$ the temperature. An elementary move consists in selecting a random monomer on one of the two strands. If the selected monomer is unbound a local flip move is attempted. If the selected monomer is a bound monomer there are two possibilities. Either a local flip of the chosen monomer is attempted, and if accepted, this move results in the bond breakage; or a flip move of both bound monomers is generated, which does not break the bond between them. We fix the rate of single monomer move to 1 and choose a rate $p_{d}$ for the double strand move. In most of the computations we took $p_{d}=1$, but we also considered different values of $p_{d}$, in particular $p_{d}<1$, which implies a reduced "mobility" for the double strands. The limiting value of $p_{d}=0$ corresponds to a double strand dynamics which can evolve only through bond breakage and which is very unlikely to happen at low temperatures. In this case the double stranded configuration remains basically "frozen" during the zipping process.

We will consider two models of zipping, as done in the study of mechanical unzipping of polymers [4]. We will refer to them as model B and model Y. In model B one has plain zipping with no constraints and the formation of bubbles (loops) is permitted. In model Y all loops are suppressed. In this case we impose the constraint that monomer $i$ can bind to its complementary only if monomer $i 1$ is already bound. In this case zipping proceeds strictly sequentially from the joining point of the two polymers to the 


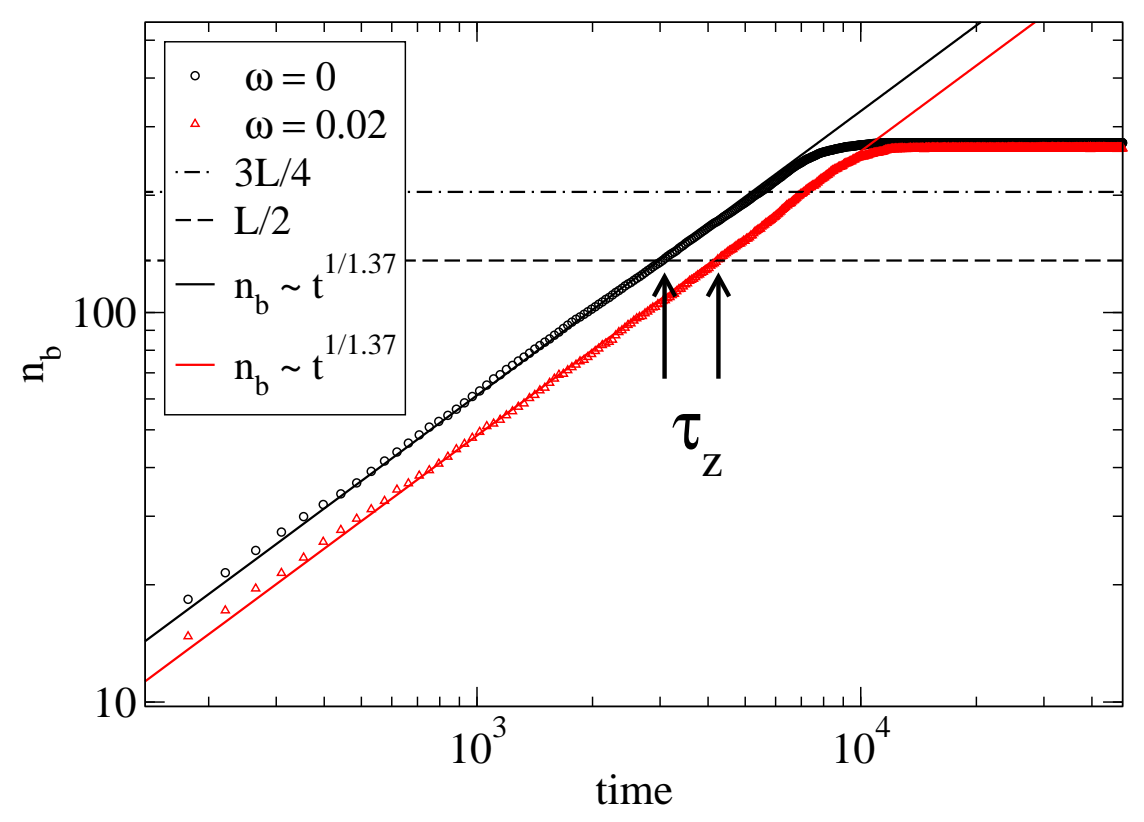

Figure 2. Number of bound monomers $n_{b}(t)$ as a function of time $t$ (measured as the number of attempted Monte Carlo moves per monomer). The length of the chains is $L=273$ and the dynamics is that denoted as model $\mathrm{B}$. The two sets of data refer to two different temperatures: $\omega=0(T=0)$ and $\omega=0.02\left(T \approx T_{c} / 2\right)$. The straight line has slope 1/1.37 and approximates well the observed power-law increase of $n_{b}(t)$. The arrows denote the values of $\tau_{z}$ obtained from $n_{b}\left(\tau_{z}, L\right)=L / 2$. Using as an alternative criterion $n_{b}\left(\tau_{z}, L\right)=3 L / 4$ (dotted-dashed line) would produce different estimates for $\tau_{z}$, which however scale with the same power-law behavior as for $L / 2$ (see text).

opposite ends of the two strands. With the flip moves used in the model two strands or part of the same strand never cross. There is however a possibility that the two strands "cut through" each other as follows. Two monomers on different strands with the same index $i$ bind to each other (by overlapping on the same lattice site) and then unbind in a different direction from the original one, which may result in a strand crossing. One can easily realize that this crossing may happen only in model B. In addition unbinding is rare at very low temperature, so the crossing may be relevant only close to the unbinding temperature. As in our analysis we do not observe differences in the scaling of the zipping times in the two models we conclude that the crossing through binding and unbinding at homologous sites is irrelevant for the universal scaling behavior of the dynamics.

\section{Results}

In the simulations we monitored the number of bound bases $n_{b}(t)$ as a function of time $t$. Figure 2 shows a plot of $n_{b}(t)$ for two different temperatures for $L=273$ and averaged over 50 different realizations. In both cases the number of bound monomers increases in time until a saturation value, corresponding to the zipping of the polymer over its 
entire length, is reached. For the scaling of $n_{b}$ we expect:

$$
n_{b}(t, L)=L f\left(t / L^{\alpha}\right)
$$

with $f()$ a scaling function. For large values of $x=t / L^{\alpha}, n_{b}$ reaches a saturation value $n_{b} \sim L$, hence $f(x) \rightarrow 1$ for $x \gg 1$. For $x \ll 1$ (short times) $n_{b}$ should be independent of $L$, hence

$$
n_{b}(t, L) \sim t^{1 / \alpha}
$$

One can thus extract the exponent governing $\alpha$ from a power-law fit of $n_{b}$ vs. $t$ in for very long polymers, to avoid finite size effects. The solid line in Fig. 2 shows that the data for $L=273$ are consistent with a value $\alpha \approx 1.37$. However, due to the presence of a saturation value one has to limit the analysis of $n_{b}(t)$ to some maximal time $t_{\max }$. In addition, the short time behavior is usually affected by deviations from the asymptotic regime (one notices some curvature of the data at early times in Fig. 2). Hence the analysis of the slope of $\log n_{b}$ vs. $\log t$ can only be performed on a limited time interval, which introduces some arbitrariness in the procedure and uncontrolled errors on the value of $\alpha$.

We used here a different approach and defined the zipping time as the time needed to reach (for the first time) a configuration where half of the monomers are bound. Figure 2 illustrates how $\tau_{z}$ is obtained from the data for the two temperatures shown. In practice, the simulations are stopped each time the number of bound monomers reaches $L / 2$, which also avoids long runs. To get an accurate estimate of $\tau_{z}$ we averaged over about $10^{3}$ independent simulations. Statistical errors are obtained from the standard deviations on these different runs.

In Fig. 3 we present a log-log plot of the zipping times as a function of the strand length in some illustrative cases in model B. We note that the asymptotic scaling sets in already at relatively short chains $(L \simeq 70)$. The data refer to different temperature values, ranging from $T=0$ to $T \approx 0.8 T_{c}$ (we estimated the critical value using equilibrium simulations with the pruned-enriched Rosenbluth method [29, 30] to be $\omega_{c} \simeq 0.1266$ ). The data are in agreement with a constant value of $\alpha$ which we summarize as $\alpha=1.38(3)$. At higher temperatures, close to the critical point (see inset, which shows $\tau_{z}$ for $T \approx 0.94 T_{c}$ for lengths up to $L=800$ ), the data show some curvature in the log-log scale, but for sufficiently long polymers they seem to approach the same exponent found in the low temperature cases (solid line). A recent paper on forced translocation [23] presented simulation evidences of the existence of two different regimes in the scaling of the translocation time as a function of $L$. At strong forcing a scaling would be governed by an exponent $\alpha \approx 1.37$, while at weak forcing by $\alpha \approx 1+\nu \approx 1.58$ [23]. In the present model we do not see a clear evidence of a second regime. However, close to $T_{c}$ the data approach the asymptotic scaling $\sim L^{1.37}$ from "below". Finite $L$ data are characterized by a higher running exponent.

We investigated the scaling of the zipping time for different values of the parameters. Figure 4 shows the case $\omega=0.02$ and compare model B with the model Y, using different values for the parameter $p_{d}$ (the mobility of the zipped part of the polymer) and using 


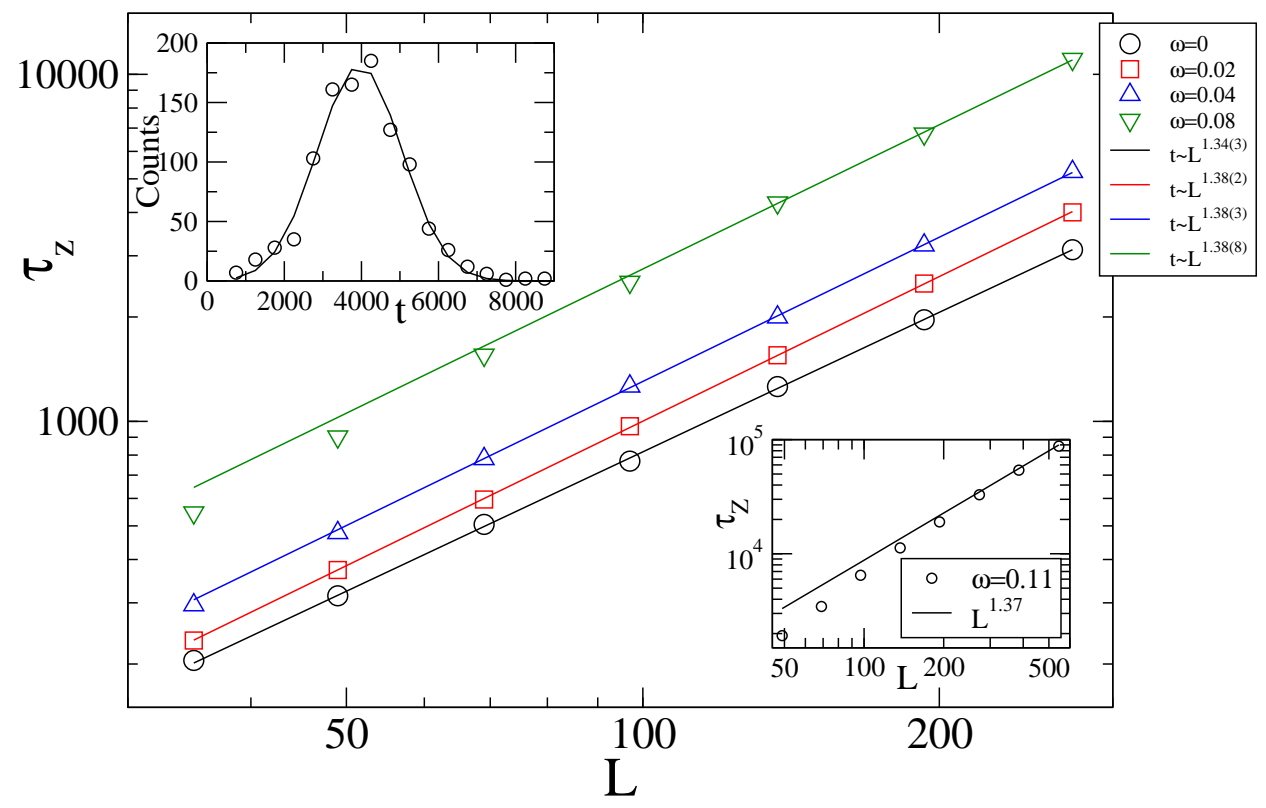

Figure 3. Log-log plots of the zipping times (model B) as a function of the chain length for $\omega=0$ ( $T=0$ or very strong bonds) in black, $\omega=0.02\left(T \simeq 0.5 T_{c}\right)$ in red, $\omega=0.04\left(T \simeq 0.67 T_{c}\right)$ in blue and $\omega=0.08\left(T \simeq 0.8 T_{c}\right)$ in green. In the last case the fit is on the last 3 points. Bottom inset: $\tau_{z}$ vs. $L$ plot for $T \approx 0.94 T_{c}$ up to $L=800$. The small $L$ data are characterized by a pronounced curvature. For sufficiently long chain the asymptotic $\sim L^{1.37}$ scaling seems to be recovered. Top inset: histogram of the zipping times for $\omega=0.02, L=273$ obtained from $10^{3}$ independent runs. The distribution of times is well-approximated by a gaussian, as shown by the fitting curve (correlation coefficient $r=0.991$ ). The error on the mean is calculated as $\delta \tau_{z}=\frac{\sigma}{\sqrt{N_{r}}} \simeq 35$, with $\sigma^{2}$ being the variance of the gaussian and $N_{r}=1000$ being the number of runs. This example shows that in this and the following graphs error bars are smaller than symbol sizes.

an alternative definition of zipping time. In the latter we used the time needed to reach a configuration where $3 L / 4$ monomers are bound for the first time. Apart from a global shift of the time scales we find that the two criteria of defining the zipping time from $n_{b}=L / 2$ or $n_{b}=3 L / 4$ yield the same value of the exponent $\alpha$. In all cases analyzed in model $\mathrm{B}$ and model $\mathrm{Y}$ the estimated exponent are consistent. Again we find as final estimate $\alpha=1.37(2)$. In the zipping dynamics and for the lattice models studied here this exponent is particularly robust and is observed in different temperature regimes and at different values of the parameters.

We turn now to the study of the dependence of the zipping time on the driving force. In the case of forced polymer translocation, the driving force is the difference in chemical potential for monomers on the two sides of the separating membrane. In our model one can view the equilibrium critical point $\left(T=T_{c}\right)$ as the limit of zero applied force. We define then the driving force as $F=\ln \left(\omega_{c} / \omega\right)$, where $\omega$ is the Boltzmann weight associated to the unbinding of two monomers and $\omega_{c}$ its value at the critical point. Figure 5 shows a plot of the zipping time for model $\mathrm{B}$ with $L=385$, as a 


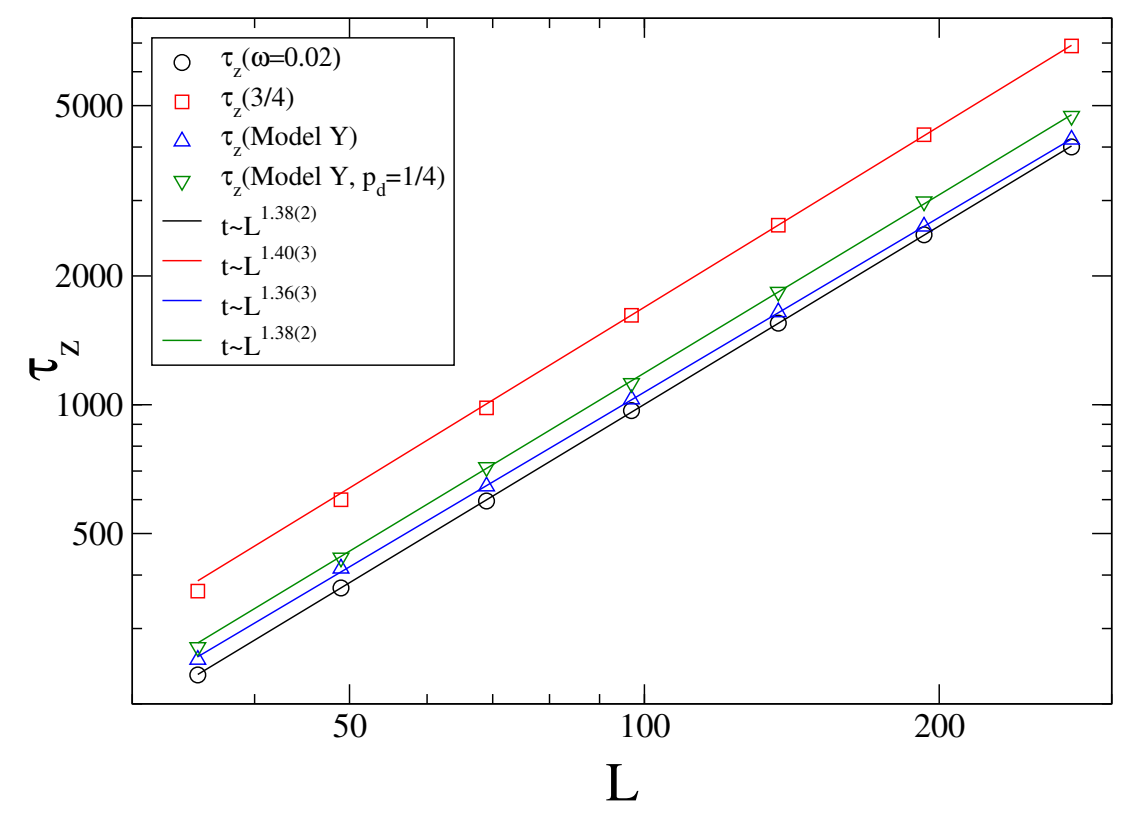

Figure 4. Log-log plots of the zipping times as functions of the polymer length for $\omega=0.02$. The four data sets correspond to four different choices of the parameters and models of the simulations. Both for models B and Y, for different values of the double stranded mobility $\left(p_{d}\right)$ and with a different criterion for the definition of the zipping time we find (the time to have $3 L / 4$ monomers bound) give a constant value for $\alpha=1.37(2)$.

function of $F$. Extrapolation of the data at weak forces $(F \leq 0.5)$ yields a scaling $\tau_{z} \sim F^{-\gamma}$ with $\gamma=0.92(5)$. This is close to the scaling $\tau_{z} \sim 1 / F$ observed in polymer translocation [13. The small deviation from the $1 / F$ scaling is likely due to the fact that the data are not in the full asymptotic regime. In addition, the shape of the $\tau_{z}$ vs. $F$ data in Fig. 5 shows a turnover from the linear response regime $\sim 1 / F$ towards a smaller slope at stronger forces which is very similar to the $\tau$ vs. $1 / F$ plot observed in translocation [23]. The dashed line in Fig. 5 has a slope $\gamma=0.8$, as observed for simulations of polymer translocaltion for for strong forces [23], beyond the linear regime. Obviously this intermediate regime is rather narrow and it is difficult to characterize it from the analysis of the simulation data. Recent analytical work [31, 32] on a simplified model of polymer translocation predicts the existence of an intermediate regime where the $\tau_{t} \sim 1 / F$ breaks down. This is consistent with our findings, however the exponents [32] do not seem to match the numerical results for the zipping dynamics. This is an interesting point which deserves further theoretical investigations. In our simulations, beyond $F \gtrsim 3$, the zipping time is weakly dependent on $F$, and the $\tau_{z}$ vs. $F$ tends towards a flat asymptotic limit at large forces. We stress that for the whole range of forces shown in Fig. 5 and up to $F \rightarrow \infty$, the scaling of $\tau_{z}$ vs. $L$ is governed by an exponent $\alpha \approx 1.37$.

To gain some more insight on the polymer conformation we investigated the radius of gyration of the two single strands and that of the double stranded part during 


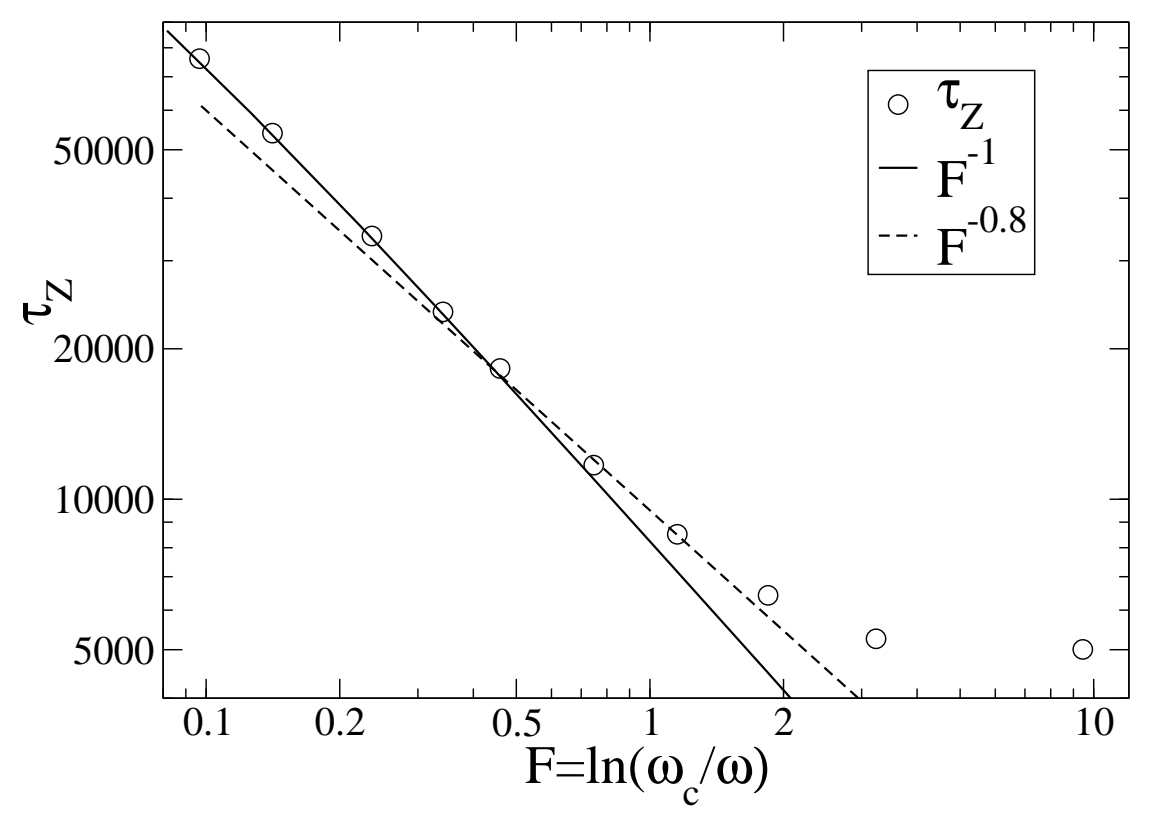

Figure 5. Plot of the unzipping time $\tau_{z}$ as a function of the parameter $\ln \left(\omega / \omega_{c}\right)$ (the driving force) for $L=385$ and model B. Here $\omega_{c}=\exp \left(\varepsilon / k_{B} T_{c}\right)$ is the value of the weight at the thermal unzipping temperature. At small forces the scaling is consistent with $\tau_{z} \sim 1 / F$. The dashed line shows a comparison with the results of Ref. [23, expected to be valid at stronger forcing.

zipping. We restricted ourselves to model $\mathrm{Y}$ in which inner loops in the zipped strand are suppressed. For polymers of length $L$ we computed the radius of gyration when each individual Monte Carlo run reaches $L / 2$ bound monomers for the first time. The average value over different independent runs is then taken. As no loops are allowed, the configuration with $L / 2$ bound monomers corresponds to that of a star polymer with three arms of length $L / 2$, each.

Figure 6 plots the radius of gyration of the single strands and of the zipped part of the polymer as a function of $L$ and in the inset that of an equilibrated star polymer with three arms of lengths $L / 2$, for a comparison. There are some differences in the scaling of the radius of gyration for the single stranded segments $\left(R_{g} \sim L^{0.59(1)}\right)$ compared with that of the zipped part $\left(R_{g} \sim L^{0.54(2)}\right)$. The latter appears to be slightly more compressed compared to the prediction from the equilibrium scaling $R_{g} \sim L^{\nu}$, with $\nu=0.588$. Luo et al. [23] discussed the scaling of the gyration radius of a translocating polymer in the regime of "fast" translocation where the translocation time scales with an exponent $\alpha \approx 1.37$. They found that for the radius of gyration after translocation scales as $R_{g} \sim L^{0.51(1)}$, which is quite consistent with our estimate $0.54(2)$ for the zipped part. However, our value does not significantly differ from the equilibrium scaling; deviations from the Flory exponent could be due to finite size correction. 


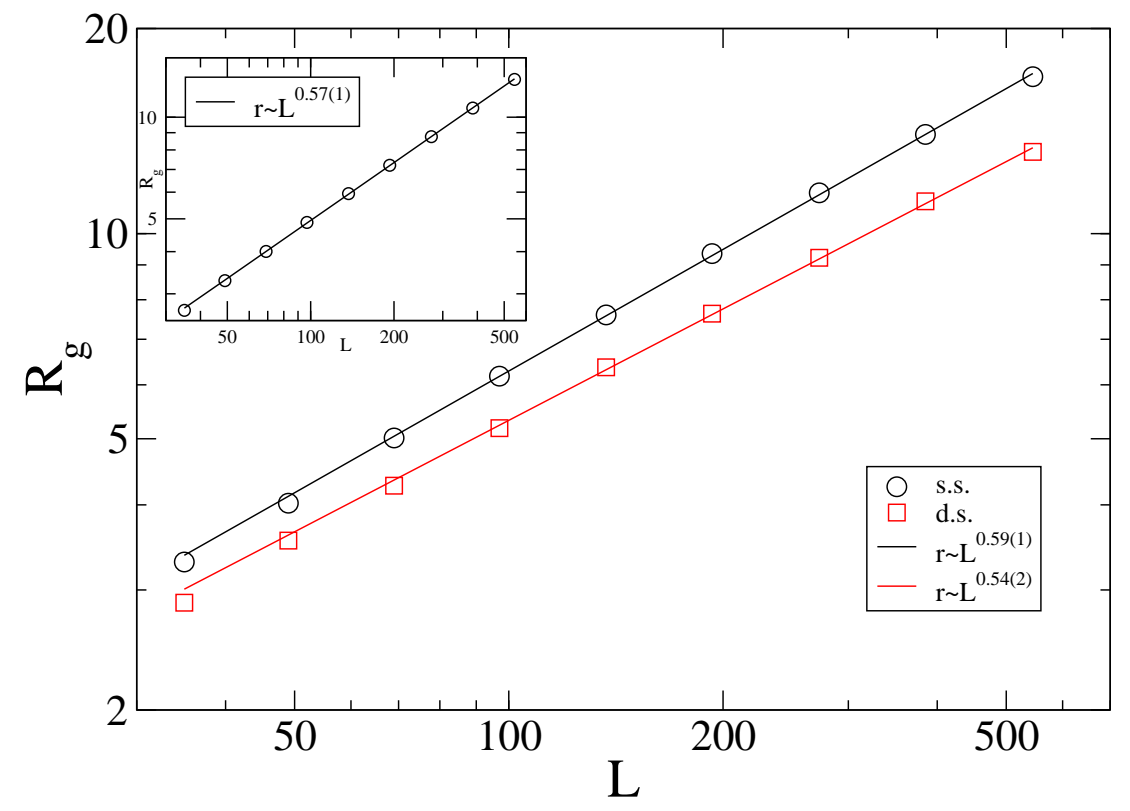

Figure 6. Plot of the radius of gyration of the single stranded segments (circles) and of the zipped part (squares) at zipping for polymers of different length. Calcualtions have been performed on the final configuration after zipping of half of the polymer in the model Y. Here $\omega=0.02$. In the inset we show a plot of the radius of gyration vs. $L$ of an equilibrated star polymer with three branches of length $L / 2$.

The local conformation of the single strands at zipping can be analyzed from the scaling of the average distance from the junction

$$
d_{Y}(n)=\frac{1}{2} \sum_{i=1}^{2}\left\langle\sqrt{\left(\vec{r}_{L / 2}^{(i)}-\vec{r}_{L / 2+n}^{(i)}\right)^{2}}\right\rangle
$$

where $\vec{r}_{k}^{(i)}$ denotes the position of the $k$-th monomer of the strand $i$, the sum is an average on the two strands and \langle\rangle denotes the average over $10^{3}$ independent Monte Carlo runs. We focus here to the case where $n$ is positive, i.e. to monomers belonging to the single strands. This quantity is plotted in Fig. 7 for strands of length $L=273$. Again the data are taken at the time when half of the bases are bound in model Y. We find that $d_{Y}(n) \sim n^{0.75(1)}$ for $n \lesssim 20$, with deviations from this scaling behavior for larger $n$, i.e. further away from the branching point. For a comparison we also plot the same quantity for an equilibrated star polymer with three arms. For the star polymer at small $n$ we find $d_{Y}(n) \sim n^{0.77(1)}$ which is indeed a similar scaling as the zipping polymer. However the polymer during zipping is "more stretched" than a star polymer at equilibrium as shown in Fig. 7. To our knowledge the scaling behavior in the vicinity of the contact point for a star polymer has not been investigate yet.

The inset of Fig. 7 shows a plot of $d_{e}(n)$ defined as

$$
d_{e}(n)=\frac{1}{2} \sum_{i=1}^{2}\left\langle\sqrt{\left(\vec{r}_{L}^{(i)}-\vec{r}_{L-n}^{(i)}\right)^{2}}\right\rangle,
$$




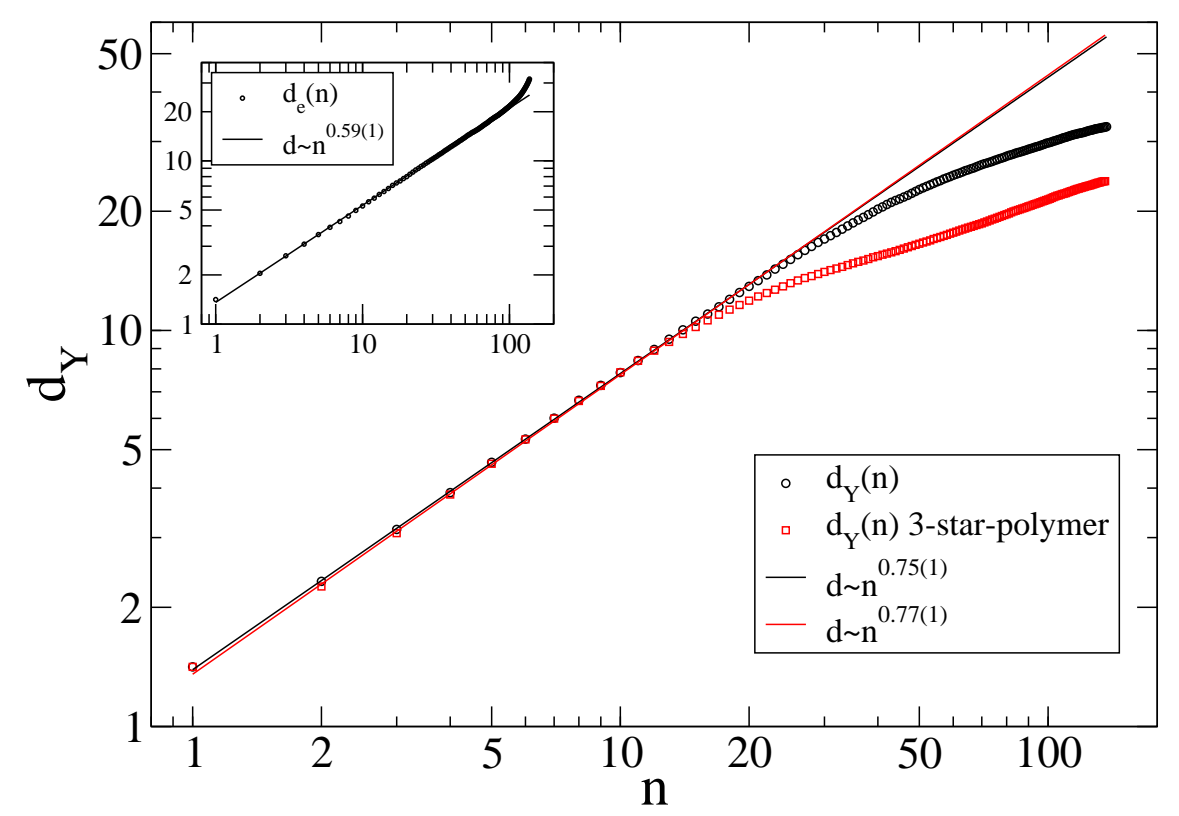

Figure 7. Plot of $d_{Y}(n)$ (Eq. (3) ) as a function of $n$. Black circles refer to the configuration of the two strands right after the zippping of half of the chains ( $L=273$, $\omega=0.02)$, while red squares refer to equilibrium configurations of a star polymer with three branches of length $L / 2$. Inset: Plot of $d_{e}(n)$ (Eq. (4)) as a function of $n$, for $L=273, \omega=0.02$. The results show that the conformation of the polymer at the far ends in in equilibrium.

which is the average distance measured from the end of the single strands. For this quantity we find a scaling in good agreement with $d_{e}(n) \sim n^{\nu}$, which implies that the local conformation close to the ends of the single strands are well-equilibrated. Thus, the analysis shows that the single strands are in a stem-flower conformation [33, 31], where the monomer density in the flower is described by the Flory exponent $\nu$. The stem is somewhat more stretched than an equilibrated star polymer, where the stretching is due to self-avoidance between the three arms which join a common contact point.

We now turn to the reverse process of unzipping. We start from an equilibrated double stranded conformation at $T=0$ and then raise the temperature to a value $T>T_{c}$. Note that, because of the double strand move incorporated in our model, a $T=0$ conformation is not frozen. A move is attempted with rate $p_{d}$. The temperature raise produces the unbinding of the two strands. In model B this is enhanced by the formation of loops proliferating along the zipped segment. In model $Y$ the contact point between the zipped strand and the single stranded segments performs a biased diffusive motion. For model Y our simulation results show that the unzipping time, defined as the average time needed to get half of the monomers unbound, scales as $\tau_{u} \sim L$. This is shown in Fig. 8, for different values of the parameter $\omega>\omega_{c}$. In this case the dynamics is thus not anomalous. 


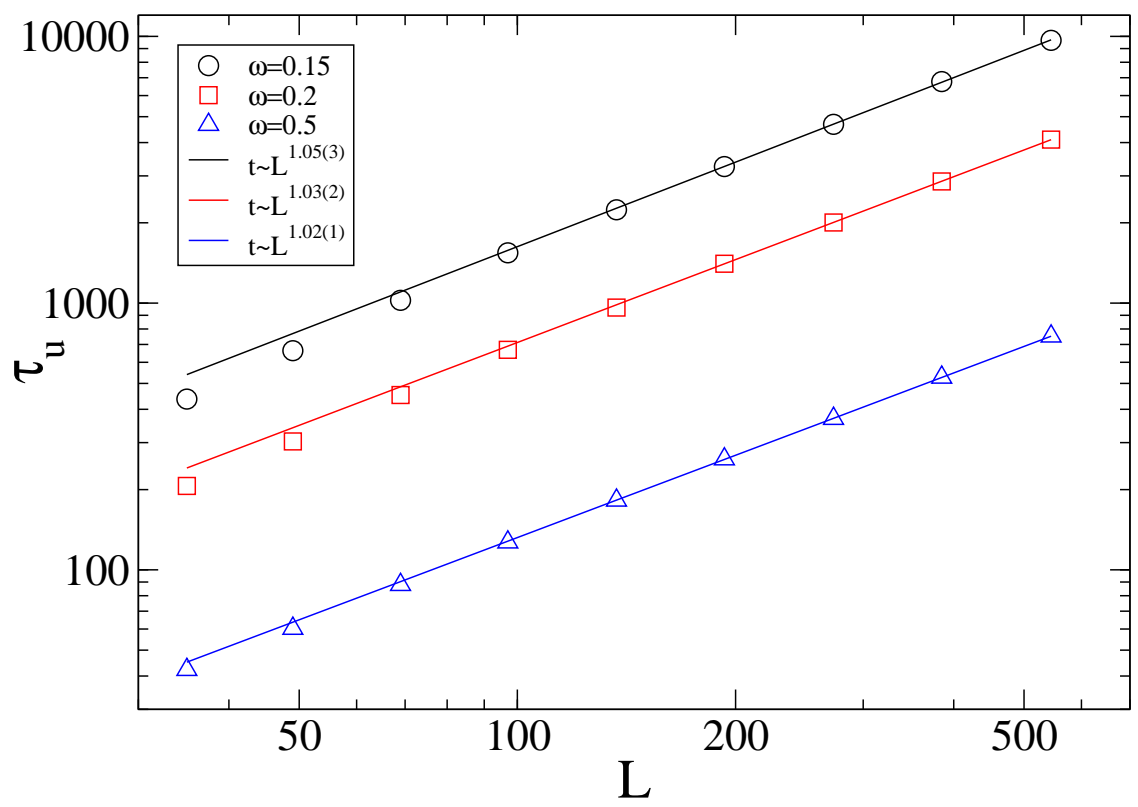

Figure 8. Plot of the unzipping time $\tau_{u}$ as a function of the chain length $L$ (model Y). Different colors stand for different values of $\omega$.

\section{Discussion}

In this paper we studied the zipping dynamics of polymers. Our numerical analysis showed that the zipping process is characterized by an anomalous exponent, with zipping times scaling as $\tau_{z} \sim L^{1.37(2)}$. Two different models were considered, with and without inner loops in the double stranded configurations. In addition we investigated a wide range of temperatures and parameters. For all the values investigated the exponent $\alpha \approx 1.37$ was confirmed. The asymptotic behavior sets in already for short polymers at low temperatures. Numerical results suggest that the scaling as a function of the driving force is $\tau_{z} \sim 1 / F$ in the limit $F \rightarrow 0$. The analysis of the gyration radii, and the comparison with those of an equilibrated star polymer, shows that the polymer configuration differs somewhat to an equilibrium one: in the vicinity of the contact point between the strands a zipping polymer is more stretched than an equilibrium polymer. However the scaling of the global radii of gyration do not differ significantly from their equilibrium counterparts.

We note that the exponent found in our simulations is in good agreement with that observed in forced translocation [13], where a chemical potential difference drives a polymer to cross a membrane through a narrow pore. Also polymer absorption seems to be governed by the same type of exponent, at least for weak adsorption energies [14]. In the zipping process discussed in this paper the polymer does not cross any planar surface and it is driven by monomer-monomer binding to close up and form a double stranded conformation. Apart from geometrical differences there are strong analogies (see Fig. 9) between zipping and translocation and it is very likely indeed that they share the same type of anomalous dynamics. If this is the case, the exponent $\alpha$ governing 


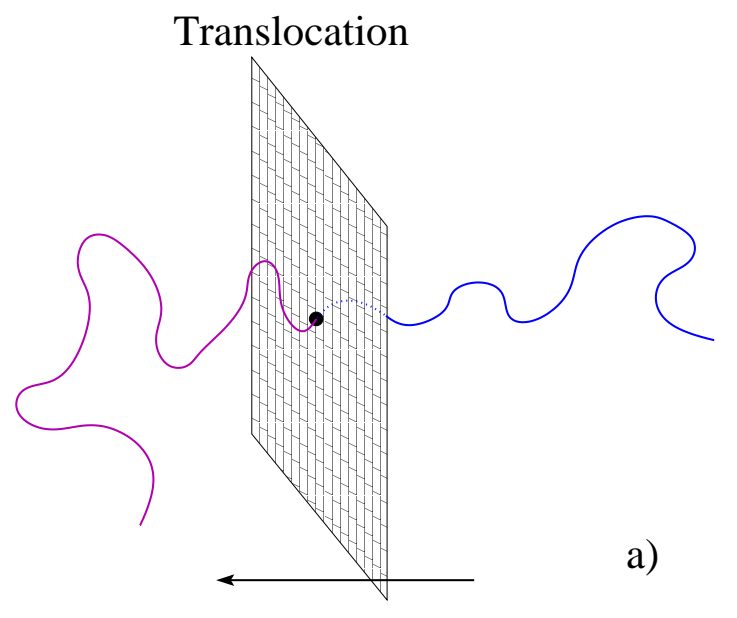

\section{Zipping}

Figure 9. Analogies between forced polymer translocation (a) and polymer zipping (b). Translocation is biased from the right side to the left side of the separating membrane. As a result the size of the left portion of the polymer increases with time. At temperatures below the unbinding temperatures, the zipped configuration (thick strands) is favored over the unzipped one (thin polymer strands). As a result the size of the zipped part increases in time. Both processes are described by similar free energies, which for the leading orders are for translocation $F(s)=\mu_{+} s+\mu_{-}(L-s)(s$ is the length of the side of the polymer, $\mu_{+}$and $\mu_{-}$the chemical potentials at the two sides), while for zipping $F(s)=f_{z} s+2 f_{u}(L-s)$ ( $s$ is the length of the zipped strand, $f_{z}$ and $f_{u}$ the free energies per unit of length of the zipped and unzipped strands). In both cases the dynamics is anomalous.

the anomalous scaling is not influenced by the geometry of the problem, i.e. whether there is a plane (as in translocation and adsorption) or a three-polymer contact point (in zipping).

A recent theory for translocation [13] predicts $\alpha=(1+2 \nu) /(1+\nu) \approx 1.37$ and $\tau_{t} \sim 1 / F$. This theory is valid at weak forcing as it assumes that the polymer conformation is not different from that in absence of driving. It is based on memory effects, which arise due to the presence of a chain tension imbalance in the vicinity of the pore in the case of translocation. In the case of zipping, we indeed observed some chain tension in the vicinity of the contact point, which causes a stretched "stem" configuration discussed above. In the case of unzipping no tension is present. The excess of monomers leading to a non-equilibrium conformation during unzipping is dissipated in other directions and does not influence the contact point dynamics. This supports indeed the idea that imbalance in the chain tension is the origin of the anomalous exponent. At stronger forcing we find that the zipping time scales non-linearly as a function of $F^{-1}$, in a similar way as found in simulations of forced translocation [23]. To our knowledge this intermediate regime is still poorly understood. Recent theoretical work [32] highlighted the complexity of the problem and predicted the existence of various translocation regimes. It is still unclear if this scenario is also valid for the zipping process.

Finally, systems where the effects discussed in this paper could be experimentally 
investigated are DNA or RNA hairpins. These are single stranded molecules which are composed by two self-complementary halfs. The self-complementarity drives the formation of a double-helical conformation at low temperatures, eventually terminating with a loop. Various aspects of the kinetics of DNA hairpins formation have been investigated (see e.g. [34, 35]), but, to our knowledge, not the length dependence of the opening/closing dynamics. Our results suggest that the scaling as a function of the sequence length of the zipping and unzipping times would be different, where only the first of them would be governed by anomalous dynamics. This is of course in absence of hydrodynamic interactions, and neglecting the winding dynamics. The latter may slow down the dynamics even further, as shown in a recent work [36].

\section{Acknowledgements:}

We acknowledge M. Baiesi, G.T. Barkema and D. Panja for useful discussions.

We acknowledge financial support from Research Foundation Flanders, Fonds voor Wetenschappelijk Onderzoek (FWO), grant G.0311.08

\section{References}

[1] D. K. Lubensky and D. R. Nelson. Pulling pinned polymers and unzipping DNA. Phys. Rev. Lett., 85:1572-1575, 2000.

[2] S. M. Bhattacharjee. Unzipping DNAs: towards the first step of replication. J. Phys. A: Math. Gen., 33:L423, 2000.

[3] K. L. Sebastian. Pulling a polymer out of a potential well and the mechanical unzipping of DNA. Phys. Rev. E, 62:1128-1132, 2000.

[4] D. Marenduzzo, S. M. Bhattacharjee, A. Maritan, E. Orlandini, and F. Seno. Dynamical scaling of the DNA unzipping transition. Phys. Rev. Lett., 88:028102, 2001.

[5] J. Mathé, A. Arinstein, Y. Rabin, and A. Meller. Equilibrium and irreversible unzipping of DNA in a nanopore. Europhys. Lett., 73:128, 2006.

[6] S. Kumar and M.S. Li. Biomolecules under mechanical force. Phys. Rep., 486:1, 2010.

[7] B. Essevaz-Roulet, U. Bockelmann, and F. Heslot. Mechanical separation of complementary strands of DNA. PNAS, 94:11935, 1997.

[8] S. Cocco, R. Monasson, and J. F. Marko. Force and kinetic barriers to unzipping of the DNA double helix. Proc. Natl Acad. Sci. U.S.A., 98:8608, 2001.

[9] S. Kumar and Y. Singh. Interpenetration and segregation of interacting polymer chains in a solution: Exact results on fractal lattices. Phys. Rev. E, 51:579, 1995.

[10] M. Baiesi, E. Carlon, E. Orlandini, and A. L. Stella. Zipping and collapse of diblock copolymers. Phys. Rev. E, 63:041801, 2001.

[11] M. Baiesi, E. Carlon, and A. L. Stella. Scaling in DNA unzipping models: denaturated loops and end-segments as branches of a block copolymer network. Phys. Rev. E, 66:021804, 2002.

[12] P. Leoni and C. Vanderzande. Statistical mechanics of RNA folding: a lattice approach. Phys. Rev. E, 68:051904, 2003.

[13] H. Vocks, D. Panja, G. T. Barkema, and R. C. Ball. Pore-blockade times for field-driven polymer translocation. J. Phys.: Condens. Matter, 20:095224, 2008.

[14] D. Panja, G. T. Barkema, and A. B. Kolomeisky. Non-equilibrium dynamics of single polymer adsorption to solid surfaces. J. Phys.: Condens. Matter, 21:242101, 2009.

[15] Y. Kantor and M. Kardar. Anomalous dynamics of forced translocation. Phys. Rev. E, 69:021806, 2004. 
[16] I. Huopaniemi, K. Luo, T. Ala-Nissila, and S.-C. Ying. Polymer translocation through a nanopore under a pulling force. Phys. Rev. E, 75:061912, 2007.

[17] M. G. Gauthier and G. W. Slater. A monte carlo algorithm to study polymer translocation through nanopores. ii. scaling laws. J. Chem. Phys., 128:205103, 2008.

[18] A. Bhattacharya and K. Binder. Out-of-equilibrium characteristics of a forced translocating chain through a nanopore. Phys. Rev. E, 81:041804, 2010.

[19] A. Bhattacharya, W.H. Morrison, K. Luo, T. Ala-Nissila, S.-C. Ying, A. Milchev and K. Binder. Scaling exponents of forced polymer translocation through a nanopore. Eur. Phys. J. E, 29:423, 2009.

[20] A. Cacciuto and E. Luijten. Confinement-Driven Translocation of a Flexible Polymer. Phys. Rev. Lett., 96:238104, 2006.

[21] I. Huopaniemi, K. Luo, T. Ala-Nissila, and S.-C. Ying. Langevin dynamics simulations of polymer translocation through nanopores. J. Chem. Phys., 125:124901, 2006.

[22] D. Panja, G. T. Barkema, and R. C. Ball. Anomalous dynamics of unbiased polymer translocation through a narrow pore. J. Phys.: Condens. Matter, 19:432202, 2007.

[23] K. Luo, T. Ala-Nissila, S.-C. Ying, and R. Metzler. Driven polymer translocation through nanopores: Slow-vs.fast dynamics. Europhys. Lett., 88:68006, 2009.

[24] J. F. Douglas, H. M. Schneider, P. Frantz, R. Lipman, and S. Granick. The origin and characterization of conformational heterogeneity in adsorbed polymer layers. J. Phys.: Cond. Matt., 9:7699, 1997.

[25] A. L. Ponomarev, T. D. Sewell, and C. J. Durning. Adsorption of isolated, flexible polymers onto a strongly attracting surface. Macromolecules, 33:2662-2669, 2000.

[26] R. Descas, J.-U. Sommer, and A. Blumen. Irreversible adsorption of tethered chains at substrates: Monte Carlo study. J. Chem. Phys., 124(9):94701, 2006.

[27] S. Bhattacharya, A. Milchev, V. G. Rostiashvili, A. Y. Grosberg, and T. A. Vilgis. Adsorption kinetics of a single polymer on a solid plane. Phys. Rev. E, 77:061603, 2008.

[28] A. Ferrantini, M. Baiesi, and E. Carlon. A lattice polymer study of DNA renaturation dynamics. J. Stat. Mech.: Theory and Exp., 2010:P03017, 2010.

[29] T. Garel and H. Orland. Guided replication of random chain: a new Monte Carlo method. J. Phys. A: Math. Gen., 23:L621, 1990.

[30] P. Grassberger. Pruned-enriched Rosenbluth method: Simulations of $\theta$ polymers of chain length up to 1000 000. Phys. Rev. E, 56:3682-3693, 1997.

[31] T. Sakaue. Nonequilibrium dynamics of polymer translocation and straightening. Phys. Rev. E, 76:021803, 2007.

[32] T. Sakaue. Sucking genes into pores: insight into driven translocation. Phys. Rev. E, 81:041808, 2010.

[33] F. Brochard-Wyart. Deformations of one tethered chain in strong flows. Europhys. Lett., 23:105, 1993.

[34] G. Bonnet, O. Krichevsky, and A. Libchaber. Kinetics of conformational fluctuations in dna hairpin-loops. PNAS, 95(15):8602, 1998.

[35] J. Kim, S. Doose, H. Neuweiler, and M. Sauer. The initial step of DNA hairpin folding: a kinetic analysis using fluorescence correlation spectroscopy. Nucleic Acids Res., 34(9):2516-2527, 2006.

[36] M. Baiesi, G. T. Barkema, E. Carlon, and D. Panja. Unwinding dynamics of double-stranded polymers. J. Chem. Phys., 133:154907, 2010. 